\title{
CODIMENSION 1 ORBITS AND SEMI-INVARIANTS FOR THE REPRESENTATIONS OF AN ORIENTED GRAPH OF TYPE $\Theta_{n}$ \\ BY
}

S. ABEASIS ${ }^{1}$

\begin{abstract}
We consider the Dynkin diagram $Q_{n}$ with an arbitrary orientation $\Omega$. For a given dimension $d=\left(d_{1}, \ldots, d_{n}\right)$ we consider the corresponding variety $L_{d}$ of all the representations of $\left(Q_{n}, \Omega\right)$ on which a group $G_{d}$ acts naturally. In this paper we determine the maximal orbit and the codim.l orbits of this action, giving explicitly their decomposition in terms of the irreducible representations of $\mathcal{U}_{n}$. We also deduce a set of algebraically independent semi-invariant polynomials which generate the ring of semi-invariants.
\end{abstract}

0. Introduction. Let us consider the Dynkin diagram $\mathbb{Q}_{n}$, where we denote by $\Gamma_{0}=\{1,2, \ldots, n\}$ the set of its vertices and by $\Gamma_{1}$ the set of its edges:

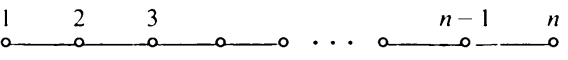

and let $\Omega$ be an orientation for its edges.

For any $d=\left(d_{1}, d_{2}, \ldots, d_{n}\right), d_{i}$ nonnegative integers, let $V_{i}$ be a vector space of dimension $d_{i}$ over a given field $K$ and let us denote by

$$
L_{d}:=L_{d}\left(\Theta_{n}, \Omega\right)=\prod_{l \in \Gamma_{1}} \operatorname{Hom}_{K}\left(V_{i(l)}, V_{f(l)}\right),
$$

where $i(l), f(l) \in \Gamma_{0}$ are respectively the initial and the final vertex of $l$ with respect to the given orientation $\Omega$. An element $B \in L_{d}$ is called a representation of the oriented graph $\left(\psi_{n}, \Omega\right)$ of dimension $d$.

The connected algebraic group $G_{d}:=\prod_{i=1}^{n} \operatorname{GL}\left(V_{i}\right)$ acts naturally on the vector space $L_{d}$, i.e. we have a rational representation $\rho: G_{d} \rightarrow \operatorname{GL}\left(L_{d}\right)$.

For any $B \in L_{d}$ let $O_{B}:=G_{d} \cdot B$ denote the orbit of $B$ and $[B]$ the set of all representations of $\left(Q_{n}, \Omega\right)$ isomorphic to $B$. Then $O_{B}=[B]$ and therefore the given action of $G_{d}$ has finitely many orbits and there is a unique maximal orbit $O_{\max } \subset L_{d}$ (open dense) (cf. $[3,5])$.

Let $\theta_{1}:=\left\{O_{B} \subset L_{d}, \operatorname{codim}_{L_{d}} \bar{O}_{B}=1\right\}$, where $\bar{O}_{B}$ is the Zariski closure of $O_{B}$.

In this paper we classify the orbits $O_{B} \in \Theta_{1}$, giving for each of them the explicit description of the indecomposable representations of $\left(Q_{n}, \Omega\right)$ which appear as factors in $[B]$. To get this result we first describe in a combinatorial way the canonical decomposition of the dimension $d$ relative to the given oriented graph (cf. [7]), i.e. we

Received by the editors April 2, 1982.

1980 Mathematics Subject Classification. Primary 14L30; Secondary 14D25.

Key words and phrases. Dynkin diagrams, representations; orbits and semi-invariants.

'The author belongs to the group GNSAGA of the C.N.R. 
describe for any $d$ the set of indecomposable factors in the maximal orbit $O_{\max } \subset L_{d}$ (cf. §3). Then the classification of the codimension 1 orbit is explicitly given in Theorem 5.1.

More precisely for any $d=\left(d_{1}, d_{2}, \ldots, d_{n}\right)$ we define a subset $I_{d}$ of pairs of indices $(i, j), i<j, i, j=1, \ldots, n$, such that the corresponding nonnegative integers $\left(d_{i}, d_{i+1}, \ldots, d_{j}\right)$ satisfy the set of inequalities and the equality stated in (4.1). Then we find a bijection $\psi: I_{d} \rightarrow \mathcal{O}_{1}, \psi(i, j)=O_{B_{i}}$, where $B_{i j}$ is explicitly constructed in terms of its indecomposable factors.

As a consequence of the properties (i) and (ii) which we establish for $\psi$ in Theorem 5.1 , we deduce for any $O_{B_{i}} \subset \mathcal{O}_{1}$ the equation for the corresponding algebraic variety $\bar{O}_{B_{i}}$; we denote this by $D_{i j}=0,(i, j) \in I_{d}$ (cf. §6).

Let us recall now that as $G_{d}$ acts on $L_{d}$ then it acts on its polynomial ring $K\left[L_{d}\right]$, and $f \in K\left[L_{d}\right]$ is a semi-invariant polynomial (or relative invariant) if $f^{g}=\chi(g) f$ for any $g \in G_{d}, \chi$ a character.

Let $\mathcal{R}_{d}:=\mathscr{G}_{d}\left(Q_{n}, \Omega\right)$ denote the ring of semi-invariants, i.e. the ring generated by the semi-invariant polynomials.

As a consequence of Theorem 5.1 and a result due to Sato and Kimura (cf. [9] and $\S 6)$, we get the following

THEOREM 0.1. If $K$ is an infinite field then:

(1) The $D_{i j}$ 's $(i, j) \in I_{d}$, are a set of algebraically independent semi-invariant polynomials.

(2) Any semi-invariant is a product of the $D_{i j}$ 's and $\Re_{d}=K\left[D_{i j}\right]$.

Note that we have already established in [1] the result of Theorem 0.1 in the case the Dynkin diagram $Q_{n}$ is equioriented. On the other side one can keep control of what happens to the ring of semi-invariants under a change of orientation for the same graph (cf. [7, Corollary, p. 77]), as any orientation $\Omega$ can be obtained from any other $\Omega^{\prime}$ performing a suitable number of simple reflections in admissible vertices (cf. also [3]). The isomorphisms given in [7] can be made completely explicit (cf. Propositions 7.1 and 7.2), and this suggests another way of proving Theorem 0.1. This method would consist in taking the proposed semi-invariants for each orientation and proving that they in fact "correspond" according to the rules given in [7]. We will sketch this method in $\$ 7$.

1. Preliminaries. Let us consider the Dynkin diagram $\mathbb{Q}_{n}$ :

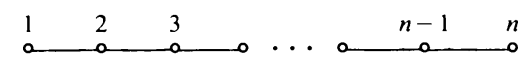

and let $\Omega$ be a given orientation for its edges. Then $\Omega$ defines the sequence $\left\{1=s_{0}<s_{1}<s_{2}<\cdots<s_{\nu}<s_{\nu+1}=n\right\}$ of vertices which are sources and sinks, the same sequence being also associated to the dual orientation $\Omega^{*}$, i.e. the orientation obtained by reversing all the arrows:

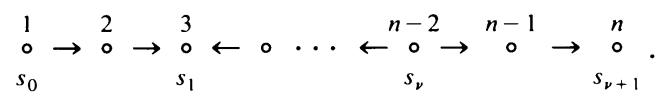

We identify $\Omega$ with the sequence $\left\{s_{i}\right\}$ as we will work up to duality and we call the $s_{i}$ 's critical points of the orientation. 
Recall that the indecomposable representations of $\left(\Theta_{n}, \Omega\right)$ are in 1-1 correspondence with the positive roots of the Dynkin diagram $Q_{n}$ independently from the orientation $\Omega$ (cf. [5 and 3]). It follows that we have an indecomposable representation $E_{p q}, p \leqslant q$, for each dimension $k=\left(k_{i}\right) \in N^{n}$ such that $k_{i}=1$ if $p \leqslant i \leqslant q$ and $k_{i}=0$ otherwise.

One can visualize the indecomposable $E_{p q}$ as an integer segment $[p, q]$ on which we have put a dot $j$ for each integer $p \leqslant j \leqslant q$, each dot $j$ representing the base vector in the corresponding one-dimensional vector space. It is also convenient to indicate the subsequence of the critical points of the orientation which are contained in $[p, q]$, i.e. $\left\{s_{i}\right\} \cap[p, q]=\left\{s_{a}, s_{a+1}, \ldots, s_{b}\right\}$, where $s_{a-1}<p \leqslant s_{a}, s_{b} \leqslant q<s_{b+1}$.

From this point of view the indecomposables $E_{p q}$ are of two types:

(1) $[p, q]$ contains an even number of critical points, and we call $E_{p q}$ or $[p, q]$ of even type.

(2) $[p, q]$ contains an odd number of critical points, and we call $E_{p q}$ or $[p, q]$ of odd type.

EXAmple. $\left(Q_{9}, \Omega\right), \Omega=\left\{s_{0}=1, s_{1}=3, s_{2}=6, s_{3}=7, s_{4}=9\right\}$.

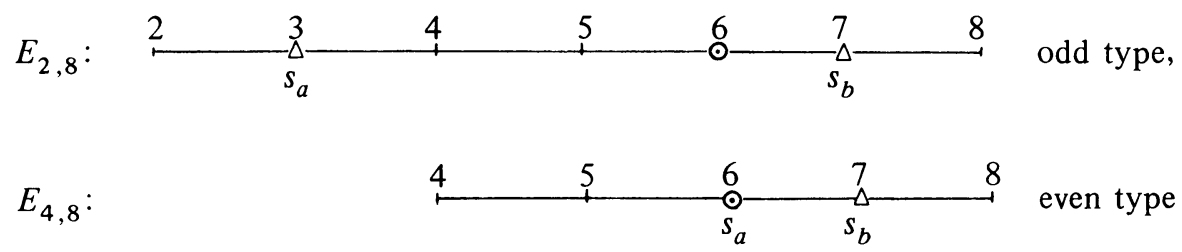

(we have denoted by $\Delta$ and $\circ$ critical points of different nature).

As soon as we know the type of the indecomposable $E_{p, q}$ and the nature of $s_{a}$, i.e. a source or a sink, we can read if the base vector $j$ is sent to $j+1$ or $j-1$ or to zero, and if $j$ is or is not in the image of $j+1$ or $j-1$.

Let $A$ be a representation of $\left(Q_{n}, \Omega\right)$. Then, up to isomorphism, it uniquely decomposes as $A=\oplus e_{p q}^{A} E_{p q}$, where $e_{p q}^{A}$ is the multiplicity of the factor $E_{p q}$. Therefore the isomorphism class of $A$, i.e. the orbit $O_{A}$, is graphically represented by a collection of "segments" $[p, q]$, each one appearing with multiplicity $e_{p q}^{A}$. We call this collection the "diagram" of $A$ or $O_{A}$.

We summarize now some results which are proved in [2]. Let $A \in L_{d}, A=\left(A_{1}\right.$, $\left.A_{2}, \ldots, A_{n-1}\right), A_{i}$ a linear map and either $A_{i}: V_{i} \rightarrow V_{i+1}$ or $A_{i}: V_{i+1} \rightarrow V_{i}$ according to the given orientation $\Omega$ of the edge $(i, i+1)$. Let $\bar{A}_{p, t}, p=t-1, t+1$, be the composition of the maps $A_{i}$ going from the sources $s_{t-1}$ or $s_{t+1}$ to the sink $s_{t}$.

For each pair of integers $u<v$ we have defined the map

$$
\varphi_{u v}^{A}: \bigoplus_{r} V_{r} \rightarrow \bigoplus_{s} V_{s}
$$

where $r$ runs over all the sources and $s$ over all the sinks of the induced oriented graph starting at $u$ and ending at $v$, whose components are

$$
V_{s_{t-1}} \oplus V_{s_{t+1}} \rightarrow V_{s_{t}}, \quad\left(z, z^{\prime}\right) \mapsto\left(\overline{A_{t-1, t}} z-\overline{A_{t+1, t}} z^{\prime}\right)
$$


Then we have set

$$
N_{u v}^{A}:= \begin{cases}\operatorname{rk} \varphi_{u v}^{A} & \text { if } u<v, \\ \operatorname{dim} V_{u} & \text { if } u=v .\end{cases}
$$

We have also defined two types of "elementary degenerations" which we can perform on pairs of indecomposables appearing as factors of a representation $A$. Let us consider integers $h<r \leqslant t<k$. Then the operations are:

(e) $E_{h k} \oplus E_{r t} \mapsto E_{h t} \oplus E_{r k},[r, t]$ of even type.

(o) $E_{h t} \oplus E_{r k} \mapsto E_{h k} \oplus E_{r t},[r, t]$ of odd type.

Note that in case (e) we also allow $r=t+1$ and we read $E_{t+1, t}$ as the zero representation, i.e. we also consider the elementary degeneration $E_{h k}=E_{h k} \oplus E_{t+1, t}$ $\mapsto E_{h t} \oplus E_{t+1, k}$.

In [2] we have proved that if $B$ is obtained from $A$ by performing on a pair of its indecomposables an operation of type (e) or (o) then $O_{B} \subset \bar{O}_{A}$ and $N_{u v}^{B} \leqslant N_{u v}^{A}$ for all $u<v$, moreover if we have the strict inequality then $N_{u v}^{B}=N_{u v}^{A}-1$. (In fact in [2] we have proved a much stronger statement which we do not need here, namely $O_{B} \subset \bar{O}_{A}$ if and only if $B$ is obtained from $A$ via a finite number of elementary operations, and if and only if $N_{u v}^{B} \leqslant N_{u v}^{A}$ for all $u<v$.) The pairs of indices $(u, v)$ for which we have $N_{u v}^{B}=N_{u v}^{A}-1$ depend on the type of the indecomposables of $A$ on which we perform the elementary degeneration (cf. [2, Proposition 4.3]). We have essentially four possible different cases which only depend on the type (odd or even) for the segments $[h, r-1]$ and $[t+1, k]$. In fact if we prescribe the type of the elementary degeneration and the type of $[h, r-1]$ and $[t+1, k]$ then we can deduce the type of each one of the two indecomposables.

EXAMPLE. $[h, r-1]$ odd, $[t+1, k]$ even correspond to the following situations:

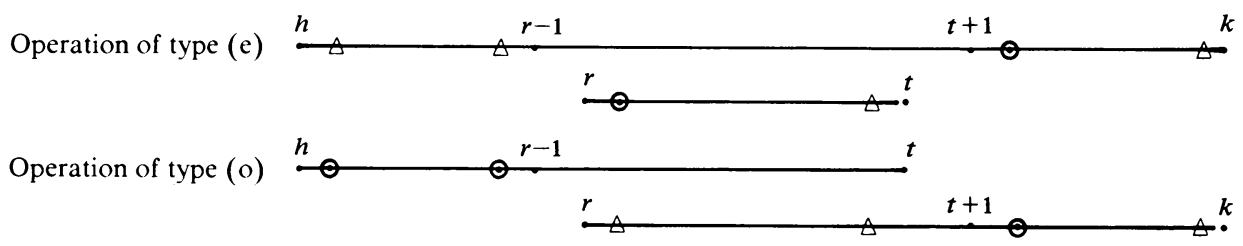

In any of these cases both $u$ and $v$ belong to a subset which is a union of integer intervals. We list these subsets in the second column of the table at the end of the next section.

Assume a representation $A$ of $\left(\mathbb{Q}_{n}, \Omega\right)$ is given through its diagram, i.e. through the decomposition $A=\oplus e_{p q}^{A} E_{p q}$. We want to show how, from the interpretation of the indecomposables as segments, we can read conditions in order to have $\varphi_{u v}^{A}$ an isomorphism for a given pair $u<v, s_{\alpha-1} \leqslant u<s_{\alpha}, s_{\beta}<v \leqslant s_{\beta+1}$. Without loss of generality we can assume $s_{\alpha}$ to be a sink, i.e. $u$ to be a source in the induced representation for the subgraph of $\mathbb{Q}_{n}$ relative to the vertices $\{u, u+1, \ldots, v\}$ (if not we can consider the dual orientation $\Omega^{*}$ ). 
LeMma 1.1. A basis for $\operatorname{ker} \varphi_{u v}^{A}$ can be given by vectors taken each from a factor $E_{p_{q}}$ in $A$ such that the subsequence of the sequence of sources and $\operatorname{sinks}\left\{u, s_{\alpha}, s_{\alpha+1}, \ldots, s_{\beta}\right.$, $v$ ) contained in $[p, q]$ starts and ends with a source.

Proof. Assume in $A$ there is a factor $E_{p q}$ such that $u<p<q<v, s_{c-1}<p \leqslant s_{c}$, $s_{d} \leqslant q<s_{d+1}, s_{\alpha-1} \leqslant u<s_{\alpha}, s_{\beta}<u \leqslant s_{\beta+1}$, and $s_{c}, s_{d}$ sources:

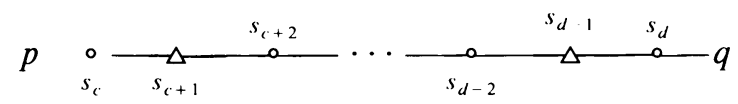

Denote by $w_{c}, w_{c+1}, w_{c+2}, \ldots, w_{d-1}, w_{d}$ respectively the base vectors in $V_{s_{c}}, V_{s_{c+1}}$, $V_{s_{c+2}}, \ldots, V_{s_{d}, 1}, V_{s_{d}}$ corresponding to the dots with same indices on the segment $[p, q]$. Then the vector $w=\left(0, \ldots, w_{c}, w_{c+2}, \ldots, w_{d-2}, w_{d}, \ldots, 0\right)$ is a nonzero vector in the space $\oplus_{r} V_{r}$, where $r$ runs over all the sources in the induced representation between $u$ and $v$. Then $w \in \operatorname{ker} \varphi_{u v}^{A}$, as follows immediately from the definition of the map $\varphi_{u v}^{A}$. The argument is similar in the other cases. Conversely with a suitable choice of the bases in the vector spaces $V_{i}$ one can always construct a basis in $\operatorname{ker} \varphi_{u v}^{A}$ as indicated before.

Using Lemma 1.1 and duality one can read from the diagram of a given representation $A$ of $\left(Q_{n}, \Omega\right)$ if $\varphi_{u v}^{A}$ is injective or surjective for any $u, v$.

EXAMPLE.

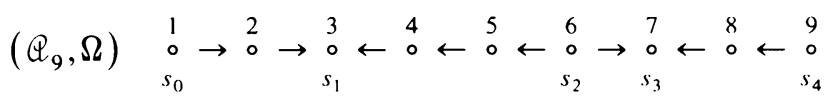

$d=(4,3,5,4,5,6,4,3,4), A \in L_{d}$,

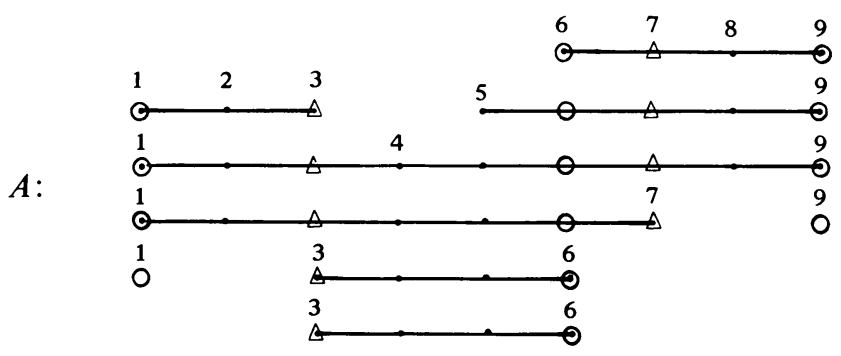

$A=E_{13} \oplus E_{19} \oplus E_{17} \oplus E_{11} \oplus 2 E_{36} \oplus E_{59} \oplus E_{69} \oplus E_{99}$,

$\varphi_{23}^{A}$ is injective but not surjective and $\operatorname{dim} \operatorname{coker} \varphi_{23}^{A}=2$,

$\varphi_{26}^{A}$ is surjective but not injective and $\operatorname{dim} \operatorname{ker} \varphi_{26}^{A}=4$,

$\varphi_{27}^{A}$ is an isomorphism since $\operatorname{dim} \operatorname{ker} \varphi_{27}^{A}=\operatorname{dim} \operatorname{coker} \varphi_{27}^{A}=0$.

2. Stabilizer and codimension formulas.

Stabilizer formulas. Let $A$ be a representation of $\left(\mathbb{Q}_{n}, \Omega\right)$ of dimension $d$, then

$$
\operatorname{dim} O_{A}=\operatorname{dim} G_{d}-\operatorname{dim} \mathrm{St} A,
$$


where St $A$ is the subgroup of $G_{d}$ which fixes $A$. Moreover $\operatorname{dim} \operatorname{St} A$ is equal to the dimension of the Lie algebra of $\mathrm{St} A$, i.e.

$$
\operatorname{dim} \mathrm{St} A=\sum_{p, q, r, t} e_{p q}^{A} e_{r t}^{A} \operatorname{dim} \operatorname{Hom}_{K}\left(E_{p q}, E_{r t}\right),
$$

where $\operatorname{Hom}_{K}\left(E_{p q}, E_{r t}\right)$ is either zero or isomorphic to $K$, depending on the integers $p, q, r, t$ and the orientation $\Omega$. In any case it is easy to check that if $E_{p q} \neq E_{r t}$ and $\operatorname{Hom}_{K}\left(E_{p q}, E_{r t}\right) \simeq K$ then $\operatorname{Hom}_{K}\left(E_{r t}, E_{p q}\right)=0$, moreover $\operatorname{Hom}_{K}\left(E_{p q}, E_{p q}\right) \simeq K$.

Let $\left\langle E_{p q}, E_{r t}\right\rangle_{\Omega}:=\operatorname{Max}\left\{\operatorname{dim}_{\operatorname{Hom}}\left(E_{p q}, E_{r t}\right), \operatorname{dim}_{\operatorname{Hom}_{K}}\left(E_{r t}, E_{p q}\right)\right\}$. It is immediately seen that $\left\langle E_{p q}, E_{r t}\right\rangle_{\Omega}=\left\langle E_{p q}, E_{r t}\right\rangle_{\Omega^{*}}$ and

$$
\begin{cases}\left\langle E_{p q}, E_{r t}\right\rangle_{\Omega}=1 & \text { if } p=r \text { or } q=s \text { or } \\ & p<r \leqslant q<t \text { and }[r, q] \text { is of even type, or } \\ & p<r \leqslant t<q \text { and }[r, t] \text { is of odd type, } \\ \left\langle E_{p q}, E_{r t}\right\rangle_{\Omega}=0 & \text { otherwise. }\end{cases}
$$

Let $A=\oplus e_{p q}^{A} E_{p q}, B=\oplus e_{r t}^{B} E_{r t}$. Then we define

$$
\langle A, B\rangle_{\Omega}:=\sum_{p, q, r . t} e_{p q}^{A} e_{r t}^{B}\left\langle E_{p q}, E_{r t}\right\rangle_{\Omega}
$$

i.e. we have a bilinear form depending on the orientation $\Omega$. In particular we have

$$
\operatorname{dimSt} A=\langle A, A\rangle_{\Omega}=\sum e_{p q}^{A} e_{r t}^{A}\left\langle E_{p q}, E_{r t}\right\rangle .
$$

Codimension formulas. Let $A, B \in L_{d}\left(\Theta_{n}, \Omega\right)$. In the remaining part of this section we will assume that there exist integers $h<r \leqslant t<k$,

$$
s_{a-1}<h \leqslant s_{a}, \quad s_{c-1}<r \leqslant s_{c}, \quad s_{d} \leqslant t<s_{d+1}, \quad s_{b} \leqslant k<s_{b+1}
$$

such that either

(1) $A=E_{h k} \oplus E_{r t} \oplus \overline{A, B}=E_{h t} \oplus E_{r k} \oplus \bar{A}$, and $[r, t]$ of even type or

(2) $A=E_{h t} \oplus E_{r k} \oplus \bar{A}, B=E_{h k} \oplus E_{r t} \oplus \bar{A}$, and [r,t] of odd type,

i.e. we assume that $B$ is obtained from $A$ via an elementary degeneration of type (e) (case (1)), or of type (o) (case (2)), (in case (1) we also allow $r=t+1$, cf. $\S 1$ ).

We want to compute in both cases (1) and (2) the codimension of $\bar{O}_{B}$ in $\bar{O}_{A}$. We have $\operatorname{codim}_{\bar{O}_{A}} \bar{O}_{B}=\operatorname{dim} \mathrm{St} B-\operatorname{dim} \mathrm{St} A$, therefore we only have to compare the two stabilizers which are involved, using (2.1). It is convenient to separate the contribution given by the pairs of indecomposables in $\bar{A}$ from the one given by the remaining factors. In case (1) we have

$$
\begin{aligned}
\operatorname{dimSt} A & =\operatorname{dimSt} \bar{A}+\operatorname{dimSt}\left(E_{h k} \oplus E_{r t}\right)+\left[E_{h k} \oplus E_{r t}, \bar{A}\right] \\
& =\operatorname{dimSt} \bar{A}+2+\left[E_{h k} \oplus E_{r t}, \bar{A}\right],
\end{aligned}
$$

where $\left[E_{h k}+E_{r t}, \bar{A}\right]$ denotes the difference $\operatorname{dim} \operatorname{St} A-\operatorname{dim} \operatorname{St} \bar{A}-2$, i.e. the reciprocal contribution given to $\operatorname{dim} \operatorname{St} A$ by the factors in $E_{h k} \oplus E_{r t}$ and the factors in $\bar{A}$. Similarly

$$
\operatorname{dim} \mathrm{St} B=\operatorname{dim} \mathrm{St} \bar{A}+3+\left[E_{h t} \oplus_{r t}, \bar{A}\right] .
$$




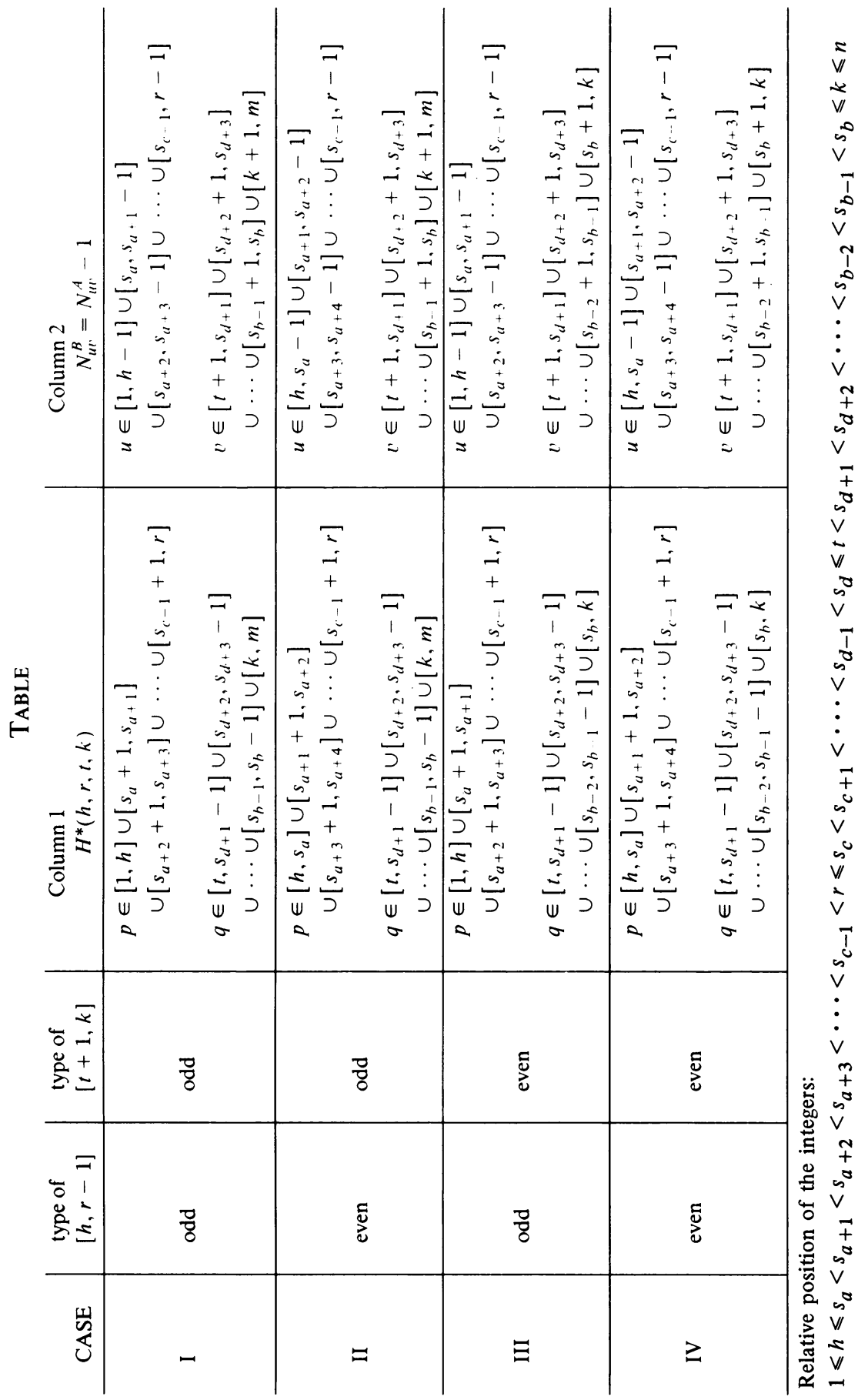


In case (2) we have similar formulas, where we have to interchange the role of $E_{h k} \oplus E_{r t}$ and $E_{h t} \oplus E_{r k}$. It follows that

$$
\begin{aligned}
\operatorname{codim}_{\overline{O_{A}}} \bar{O}_{B} & =1 \pm\left\{\left[E_{h t} \oplus E_{r k}, \bar{A}\right]-\left[E_{h k} \oplus E_{r t}, \bar{A}\right]\right\} \\
& =1 \pm \sum e_{p q}^{\bar{A}}\left\{\left[E_{h t} \oplus E_{r k}, E_{p q}\right]-\left[E_{h k} \oplus E_{r t}, E_{p q}\right]\right\}
\end{aligned}
$$

where

$$
\left[E_{u v}, E_{z w}\right]= \begin{cases}\left\langle E_{u v}, E_{z w}\right\rangle & \text { if }(u, v) \neq(z, w)(\mathrm{cf} .(2.2)), \\ 2 & \text { if }(u, v)=(z, w)\end{cases}
$$

and the sign + holds in case (1), the sign - holds in case (2). Note that $\pm\left\{\left[E_{h t} \oplus E_{r k}, \overline{A]}-\left[E_{h k} \oplus E_{r l}, \overline{A]}\right]\right\}\right.$ is in any case nonnegative, as $\bar{O}_{B} \subsetneq \bar{O}_{A}$ and therefore the codimension is at least 1.

Equation (2.4) allows us to compute explicitly $\operatorname{codim} \bar{O}_{A} \bar{O}_{B}$ once we know if we are in case (1) or (2) and we know the factors of the decomposition of $\bar{A}$.

Let us consider the set $H(h, r, t, k), h<r \leqslant t<k$ (or $r=t+1$ and $E_{t+1 . t}$ the zero representation) defined as follows:

$$
H(h, r, t, k)=\left\{(p, q):\left|\left[E_{h t} \oplus E_{r k}, E_{p q}\right]-\left[E_{h k} \oplus E_{r t}, E_{p q}\right]\right|>0\right\} .
$$

As a consequence of (2.4) we have the following

Proposition 2.5. $\operatorname{Codim}_{\bar{O}_{A}} \bar{O}_{B}=1$ if and only if for every pair $(p, q) \in H(h, r, t, k)$ we have $e_{p q}^{\bar{A}}=0$.

In order to be able to find the codim 1 orbits we need to know the set $H(h, r, t, k)$. Note that this set depends not only on the integers $h, r, t, k$ but also on the type (odd or even) of the indecomposables on which we perform the elementary degeneration (of type (e) or (o)). It is easy to see, once more, that we have four different sets corrdsponding to the type (odd or even) of the intervals $[h, r-1]$ and $[t+1, k]$.

In Column 1 of the Table we describe a set of pairs, denoted by $H^{*}(h, r, t, k)$, which is related to $H(h, r, t, k)$ as follows:

$$
\begin{cases}H(h, r, t, k)=H^{*}(h, r, t, k) \backslash\{(h, k),(r, t)\} & \text { in case }(1), \\ H(h, r, t, k)=H^{*}(h, r, t, k) \backslash\{(h, t),(r, k)\} & \text { in case }(2) .\end{cases}
$$

The sets $H$ (and therefore $H^{*}$ ) have been constructed by direct inspection using (2.2).

In Column 2 of the table we list the pairs $(u, v)$ such that $N_{u v}^{B}=N_{u v}^{A}-1, A$, $B \in L_{d}$, satisfying either (1) or (2) as we have announced in $\S 1$. We will need to compare the two columns in the proof of Theorem 5.1.

3. The canonical construction. Let $d=\left(d_{1}, d_{2}, \ldots, d_{n}\right)$ be a given dimension and $\Omega=\left\{s_{i}\right\}$ an orientation for the Dynkin diagram $\mathcal{Q}_{n}$. We describe here a construction which produces a special representation $C_{\Omega}(d) \in L_{d}\left(Q_{n}, \Omega\right)$. The main property of $C_{\Omega}(d)$ is given by the following

Proposition 3.1. Let $A:=C_{\Omega}(d)$. Then $O_{A}$ is the maximal orbit in $L_{d}\left(\mathbb{Q}_{n}, \Omega\right)$, i.e. $O_{A}=O_{\max } \subset L_{d}\left(\mathbb{Q}_{n}, \Omega\right)$. 
To describe $C_{\Omega}(d)$ we first recall the construction of the maximal orbit of a given dimension $k=\left(k_{1}, k_{2}, \ldots, k_{m}\right)$ and relative to a Dynkin diagram $Q_{m}$ which is equioriented:

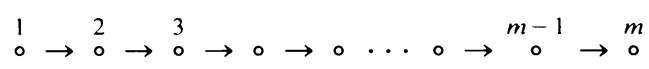

Consider an "orizontal line" $l$ on which we have the integers $1,2, \ldots, m$ and let $\pi^{+}$ be one of the two half planes determined by $l$. Draw in $\pi^{+}$on the vertical line passing through $j, 1 \leqslant j \leqslant m$, as many dots as the integer $k_{j}$, the first dot always being on the line $l$. Join with an orizontal edge, if possible, points over 1 with points over 2 , the ones over 2 with the ones over 3 , etc.

EXAMPLE. $\left(Q_{6}\right.$, equior. $), k=(2,3,4,2,5,4)$ :

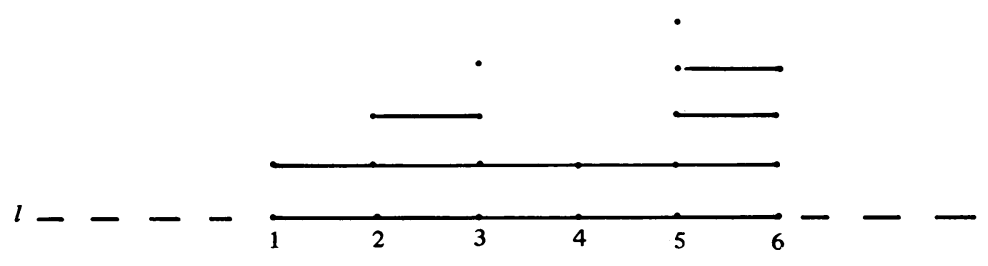

The result is a collection of "orizontal segments" which represent the indecomposable factors of any representation in the maximal orbit $O_{\max } \subset L_{k}\left(Q_{m}\right.$, equior.) (for the proof cf. [1]).

The construction $C_{\Omega}(d)$. The orientation $\Omega=\left\{1=s_{0}<s_{1}<\cdots<s_{\nu}<s_{\nu+1}=n\right\}$ determines the subgraphs $\mathbb{Q}^{\left(s_{i}\right)}$ of $\mathbb{Q}_{n}$, relative to the vertices $\left(s_{i}, s_{i}+1, \ldots, s_{i+1}\right)$, which are equioriented. The construction $C_{\Omega}(d)$ is given by steps:

(1) We construct the maximal orbit for the equioriented graph $\mathbb{Q}^{\left(s_{0}\right)}$ and relative to the dimension $d^{\left(s_{0}\right)}=\left(d_{1}, d_{2}, \ldots, d_{s_{1}}\right)$. The orizontal line is denoted by $l_{s_{0}}$.

(2) We construct the maximal orbit for the equioriented graph $\mathbb{Q}^{\left(s_{1}\right)}$ and relative to the dimension $d^{\left(s_{1}\right)}=\left(d_{s_{1}}, d_{s_{1}+1}, \ldots, d_{s_{2}}\right)$ choosing the orizontal line $l_{s_{1}}$ passing through the last vertex over $s_{1}$ and the half plane is the one containing the line $l_{s_{0}}$.

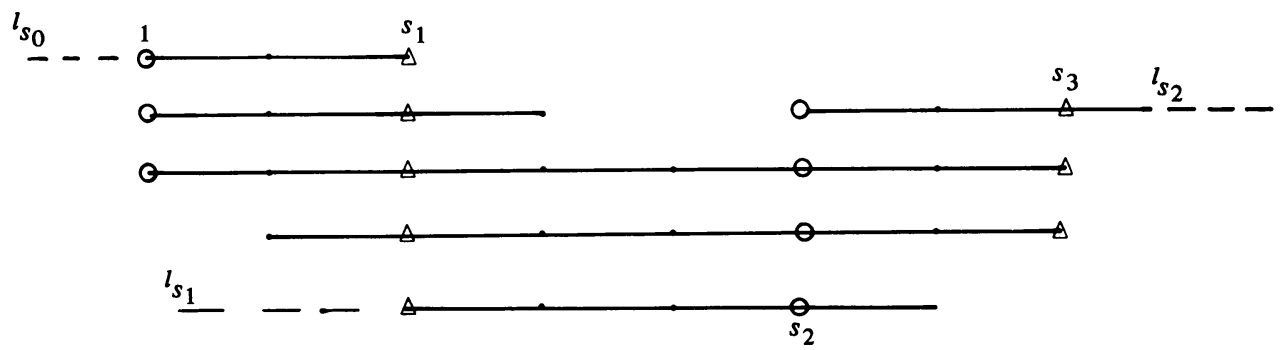

We say that we have glued together the constructions for $\mathcal{Q}^{\left(s_{0}\right)}$ and $\mathcal{Q}^{\left(s_{1}\right)}$ "reversing the orientation". 
We proceed this way constructing the maximal orbit for $\mathcal{Q}^{\left(s_{1}\right)}$ of dimension $d^{\left(s_{1}\right)}=\left(d_{s_{1}}, d_{s_{1}+1}, \ldots, d_{s_{1+1}}\right)$ and glueing it to the previous one but reversing the orientation $(i=1,2, \ldots, \nu)$. The result is a collection of indecomposables which determines an element $A:=C_{\Omega}(d) \in L_{d}\left(Q_{n}, \Omega\right)$. An example of such a construction has been given at the end of $\S 1$.

Lemma 3.2. Let $A=C_{\Omega}(d)$ and assume $E_{p q}$ appears as a factor in $A$. Then in $A$ there cannot appear any factor $E_{p^{\prime} q^{\prime}}$ such that:

(a) $p^{\prime}>p, q^{\prime}<q$ and $\left[p^{\prime}, q^{\prime}\right]$ of odd type:

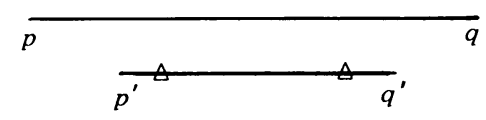

(b) $p^{\prime}<p, q^{\prime}<q$ and $\left[p, q^{\prime}\right]$ of even type:

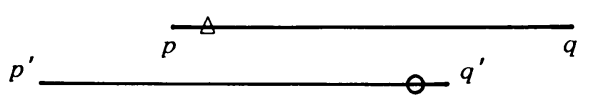

(c) $p^{\prime}>p, q^{\prime}>q$ and $\left[p^{\prime}, q\right]$ of even type:

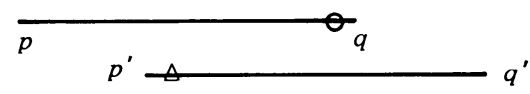

(d) $p^{\prime}<p, q^{\prime}=p-1$ or $p^{\prime}=q+1, q^{\prime}>q$ :

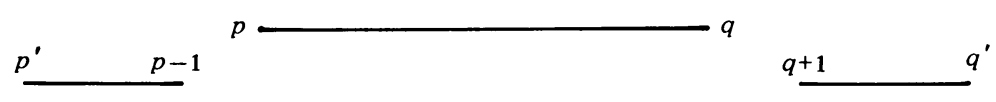

Proof. The proof follows by counting how many times one has reversed the orientation for the construction between $p^{\prime}-1$ and $q^{\prime}+1$ in case (a), between $p-1$ and $q^{\prime}+1$ in case (b), and similarly for (c) and (d).

REMARK 3.3. Let $\Omega^{*}$ be the orientation dual to $\Omega$, then:

(i) $C_{\Omega}(d)=C_{\Omega^{*}}(d)$.

$$
\operatorname{dim} \mathrm{St} A=\operatorname{dimSt} C_{\Omega}(d)=\sum_{p \leqslant q} e_{q p}^{A}\left(\sum_{t<q} e_{p t}^{A}+\sum_{t \geqslant p} e_{t q}^{A}\right) .
$$

Proof of Proposition 3.1. We only need to verify that

$$
\operatorname{dim} \operatorname{St} A=\sum_{i=1}^{n} d_{i}^{2}-\sum_{i=1}^{n-1} d_{i} d_{i+1}=d_{1}^{2}+\sum_{i=2}^{n} d_{i}\left(d_{i}-d_{i-1}\right)
$$


We proceed by induction on the length of the graph. Let $\left(Q_{n-1}, \Omega^{\prime}\right)$ be obtained from $\left(\Theta_{n}, \Omega\right)$ by dropping the last vertex $n$, where $\Omega^{\prime}=\left\{1=s_{0}<s_{1}<\cdots<s_{\nu} \leqslant n-1\right.$ $\left.=s_{\nu+1}^{\prime}\right\}$ is the orientation induced by $\Omega$ on $Q_{n-1}$. Let $d^{\prime}=\left(d_{1}, d_{2}, \ldots, d_{n-1}\right)$ and $A^{\prime}=C_{\Omega^{\prime}}\left(d^{\prime}\right) \in O_{\max }^{d^{\prime}}$ (inductive assumption). We only have to prove that

$$
\operatorname{dim} \operatorname{St} A=\operatorname{dim} \operatorname{St} A^{\prime}+d_{n}\left(d_{n}-d_{n-1}\right) .
$$

As we have $e_{p q}^{A}=e_{p q}^{A^{\prime}}$ for all $p \leqslant q<n-1$, using (2.2) and (2.1) we only have to compare the contribution given by the factors of type $E_{p, n-1}$ in $A^{\prime}$ to $\operatorname{dimSt} A^{\prime}$ with the one given by the factors of $A$ of type $E_{p n}$ and $E_{p, n-1}$ to $\operatorname{dim} \operatorname{St} A$. Note that each pair $E_{p, n-1}, E_{t, n-1}$ which appears in $A^{\prime}$ gives a contribution of 1 to $\operatorname{dimSt} A^{\prime}$ (cf. (2.1), (2.2) and also Remark 3.3(ii)). If $d_{n}=d_{n-1}$, all the factors of type $E_{p, n-1}$ in $A^{\prime}$ change to $E_{p n}$ in $A$ and $e_{n n}^{A}=0$; therefore $\operatorname{dim} \mathrm{St} A=\operatorname{dim} \mathrm{St} A^{\prime}$ and the claim is proved. If $d_{n}>d_{n-1}$, all the factors $E_{p, n-1}$ in $A^{\prime}$ change to $E_{p n}$ in $A$ and we have exactly $d_{n}-d_{n-1}$ factors $E_{n n}$ in $A$; therefore we have exactly $d_{n}\left(d_{n}-d_{n-1}\right)$ new pairs of indecomposables which give a contribution of 1 to $\operatorname{dim} \operatorname{St} A$, and the claim is proved.

If $d_{n}<d_{n-1}$ then $d_{n}$ of the factors $E_{p, n-1}(p=1, \ldots, n-1)$ appearing in $A^{\prime}$ have become $E_{p n}$ in $A$ and in $A$ there are exactly $d_{n-1}-d_{n}$ factors $E_{t, n-1}$. Using Lemma 3.2 it is immediately seen that there are $d_{n}\left(d_{n-1}-d_{n}\right)$ pairs $\left(E_{p n}, E_{t, n-1}\right)$ which do not give any contribution to $\operatorname{dim} \operatorname{St} A$ which therefore decreases with respect to $\operatorname{dim}$ St $A^{\prime}$ of $d_{n}\left(d_{n-1}-d_{n}\right)$. This ends the proof.

As a result of Proposition 3.1 we have a graphical description of the indecomposable factors appearing in the decomposition of the maximal orbit. As the graph $\mathbb{Q}_{n}$ is of finite type this is equivalent to giving a combinatorial description of the "canonical decomposition" of the dimension vector $d=\left(d_{1}, d_{2}, \ldots, d_{n}\right)$ (for this notion $\mathrm{cf}$. [7 and 8]), with respect to the oriented graph $\left(\mathbb{Q}_{n}, \Omega\right)$.

The factors and the multiplicities appearing in $A=C_{\Omega}(d)$ can be numerically described as follows. Let

$$
\begin{aligned}
& m_{1}=\min \left(d_{s_{0}}=d_{1}, d_{2}, \ldots, d_{s_{1}}\right), \quad \bar{d}_{s_{1}}=d_{s_{1}}-m_{1}, \\
& m_{2}=\min \left(m_{1}, d_{s_{1}+1}-\bar{d}_{s_{1}}, \ldots, d_{s_{2}}-\bar{d}_{s_{1}}\right), \quad \bar{d}_{s_{2}}=d_{s_{2}}-\bar{d}_{s_{1}}-m_{2}, \\
& m_{3}=\min \left(m_{2}, d_{s_{2}+1}-\bar{d}_{s_{2}}, \ldots, d_{s_{3}}-\bar{d}_{s_{2}}\right), \quad \ldots \ldots \ldots \ldots \ldots \ldots \ldots \ldots \ldots \ldots \ldots \ldots \ldots \ldots \ldots \ldots \ldots, \quad \bar{d}_{s_{k-1}}=d_{s_{k-1}}-\bar{d}_{s_{k-2}}-m_{k-1}, \\
& \left.\ldots \ldots \ldots \ldots, d_{s_{k-1}+t}-\bar{d}_{s_{k-1}}, \ldots, d_{s_{k}}-\bar{d}_{s_{k-1}}\right) .
\end{aligned}
$$

In the sequence $m_{1} \geqslant m_{2} \geqslant \cdots \geqslant m_{k} \geqslant \cdots$, let $k$ be the first integer such that $m_{k} \leqslant 0$ and let $t \geqslant 0$ be such that $d_{s_{k-1}}-\bar{d}_{s_{k-1}}>0, \ldots, d_{s_{k-1}+t}-\bar{d}_{s_{k-1}}>0$, $d_{s_{k-1}+t+1}-\bar{d}_{s_{k-1}} \leqslant 0$. Then in $A=C_{\Omega}(d)$ we have a factor of type $E_{1, q}, q=s_{k-1}+$ $t$, with multiplicity

$$
e_{1, q}^{A}=\min \left(m_{k-1}, d_{s_{k-1}+1}-\bar{d}_{s_{k-1}}, \ldots, d_{s_{k-1}+t}-\bar{d}_{s_{k-1}}\right) .
$$


This numerical procedure is immediately deduced from the graphical construction. Then we apply the same procedure to the dimension vector

$$
\left(d_{1}, d_{2}, \ldots, d_{n}\right)-e_{1, q}^{A}(\underbrace{1,1, \ldots, 1}_{q \text { times }}, 0, \ldots, 0) .
$$

EXAMPLE. $d=(11,7,9, \underset{\Delta}{4}, 10,12, \underset{\circ}{6}, 8,4,11, \underset{\Delta}{3}, 5,9, \underset{\circ}{6}, 4)$

$$
\begin{array}{ll}
m_{1}=\min (11,7,9,4)=4, & \bar{d}_{s_{1}}=4-4=0, \\
m_{2}=\min (4,10,12,6)=4, & \bar{d}_{s_{2}}=6-0-4=2, \\
m_{3}=\min (4,6,2,9,1)=1, & \bar{d}_{s_{3}}=0, \\
m_{4}=\min (1,5,9,6)=1, & \bar{d}_{s_{4}}=5, \\
m_{5}=\min (1,-1)<0 . &
\end{array}
$$

In $A$ there is a factor $E_{1,14}$ with multiplicity 1 . To find the other factors we apply the same method to the dimension

$$
(10,6,8, \underset{\Delta}{3}, 9,11,5,7,3,10,2,4,8,5,4) .
$$

4. The set $I_{d}(\Omega)$. Let $d=\left(d_{1}, d_{2}, \ldots, d_{n}\right)$ be a given dimension such that $d_{i}>0$, all $i$. We define the set $I_{d}:=I_{d}(\Omega)$ to be the set of pairs $(i, j)$ with $i<j, i$, $j=1, \ldots, n$, which satisfy the following conditions with respect to the orientation $\Omega$.

Set $s_{\alpha-1} \leqslant i<s_{\alpha}, s_{\beta}<j \leqslant s_{\beta+1}$. Then $(i, j) \in I_{d}$ if and only if

$$
\begin{cases}(-1) & \text { for every } t \text { such that } i<t \leqslant s_{\alpha}, \text { then } d_{t}>d_{i} \\ (\tau) & \text { for every } \tau=0, \ldots, \beta-\alpha-1 \text { and } t \text { such that } s_{\alpha+\tau}<t \leqslant s_{\alpha+\tau+1} \\ & \text { then } d_{t}>d_{s_{\alpha+\tau}}-d_{s_{\alpha+\tau-1}}+\cdots+(-1)^{\tau} d_{s_{\alpha}}+(-1)^{\tau+1} d_{i} \\ (\beta-\alpha) & \text { for every } t \text { such that } s_{\beta}<t<j, \text { then } \\ & d_{t}>d_{s_{\beta}}-d_{s_{\beta-1}}+\cdots+(-1)^{\beta-\alpha} d_{s}+(-1)^{\beta-\alpha+1} d_{i} \\ & \text { and } \\ & d_{j}=d_{s_{\beta}}-d_{s_{\beta-1}}+\cdots+(-1)^{\beta-\alpha} d_{s}+(-1)^{\beta-\alpha+1} d_{i} .\end{cases}
$$

The set $I_{d}$ is entirely defined in terms of the given dimension and the orientation $\Omega$. We will show soon that it has the same cardinality as the set $\theta_{1}$ of the codim 1 orbits in $L_{d}\left(Q_{n}, \Omega\right)$.

We first interpret the conditions defining $I_{d}$ in terms of the indecomposable factors of the maximal orbit, i.e. in terms of the canonical construction $C_{\Omega}(d)$. Let $A=C_{\Omega}(d) \in O_{\max }^{d}$ and $(i, j) \in I_{d}, s_{\alpha-1} \leqslant i<s_{\alpha}, s_{\beta}<j \leqslant s_{\beta+1}$. We can assume that $s_{\alpha}$ is a sink (cf. Remark 3.3(i)). Condition (-1) in (4.1) is equivalent to saying that any indecomposable factor in $A$ which contains the vertex $i$ contains also $s_{\alpha}$ (this set is not empty as $d_{i}>0$ ); there are indecomposables which start at $i+1$ and contain $s_{\alpha}$. As a consequence of Lemma 1.1 it follows that $\varphi_{i t}^{A}, i<t \leqslant s_{\alpha}$, is injective 
but not surjective and $d_{t}-d_{i}=\operatorname{dim} \operatorname{coker} \varphi_{i t}^{A}$. Condition (0) says that for every $t$, $s_{\alpha}<t \leqslant s_{\alpha+1}$, the map $\varphi_{i t}^{A}$ is surjective but not injective and $d_{t}-d_{s_{\alpha}}+d_{i}=$ $\operatorname{dim} \operatorname{ker} \varphi_{i t}^{A}$. The set of factors of $A$ which contain the vertices $i$ and $s_{\alpha+1}$ and the set of factors of $A$ which start at $i+1$ and contain $s_{\alpha+1}$ are not empty.

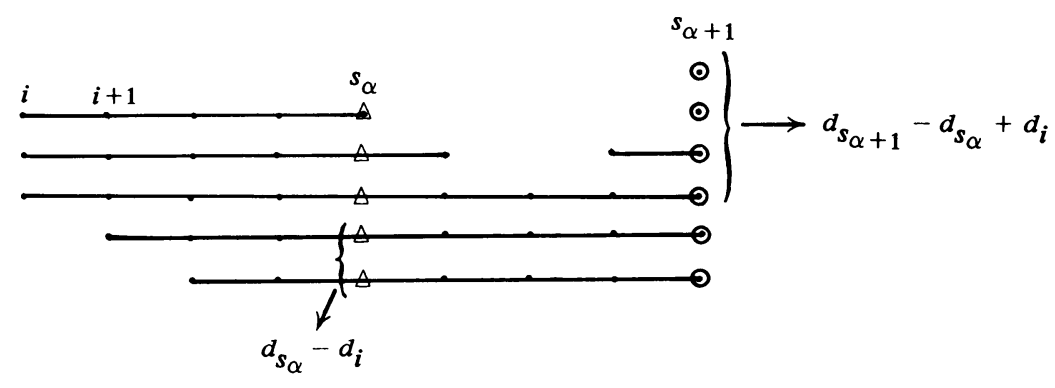

In general, condition $(\tau), \tau=0, \ldots, \beta-\alpha-1$ and $s_{\alpha+\tau}<t \leqslant s_{\alpha+\tau+1}$, or $\tau=\beta$ $-\alpha$ and $s_{\beta}<t<j$ says that:

$\varphi_{i t}^{A}$ is surjective but not injective if $\tau$ is even and

$$
d_{t}-d_{s_{\alpha+\tau}}+\cdots+d_{i}=\operatorname{dim} \operatorname{Ker} \varphi_{i t}^{A} .
$$

$\varphi_{i t}^{A}$ is injective but not surjective if $\tau$ is odd and

$$
d_{t}-d_{s_{\alpha+\tau}}+\cdots-d_{i}=\operatorname{dim} \operatorname{Coker} \varphi_{i t}^{A} .
$$

The equality in condition $(\beta-\alpha)$ says that $\varphi_{i j}^{A}$ is both injective and surjective, i.e. is an isomorphism.

REMARK 4.2. In particular, $(i, j) \in I_{d}$ implies that if $(\beta-\alpha)$ is odd then the set of factors in $A$ which contain both the vertices $i$ and $j$ is not empty, and there is at least a factor $E_{i+1, j-1}$; moreover there are no factors in $A$ which contain the vertex $i$ and end at $j-1$ or which start at $i+1$ and contain $j$. If $(\beta-\alpha)$ is even then the set of factors in $A$ which start at $i+1$ and contain $j$ and the ones which contain $i$ and end at $j-1$ are not empty; moreover in $A$ there are no factors which contain both $i$ and $j$ or which start at $i+1$ and end at $j-1$. We will use this observation to construct the injective map in Theorem 5.1.

The condition (4.1) can be rewritten as follows:

$$
\begin{cases}(-1) & \text { for every } t \text { such that } s_{\beta} \leqslant t<j, \text { then } d_{t}>d_{j} \\ (\tau) & \text { for every } \tau=0, \ldots, \beta-\alpha-1 \text { and } t \text { such that } s_{\beta-\tau-1} \leqslant t<s_{\beta-\tau} \\ & \text { then } d_{t}>d_{s_{\beta-\tau}}-d_{s_{\beta-\tau-1}}+\cdots \pm d_{j} \\ (\beta-\alpha) & \text { for every } t \text { such that } i<t<s_{\alpha}, \text { then } \\ & d_{t}>d_{s_{\alpha}}-d_{s_{\alpha+1}}+\cdots \pm d_{j} \\ & \text { and } \\ & d_{i}=d_{s_{\alpha}}-d_{s_{\alpha+1}}+\cdots \pm d_{j}\end{cases}
$$


Clearly we can read the conditions of (4.1)' in terms of the maps $\varphi_{t j}^{A}, i \leqslant t<j$, as we have done for the conditions (4.1).

Proposition 4.3. The cardinality $\left|I_{d}\right|$ of the set $I_{d}$ is $\left|I_{d}\right|=n-s$, where $n$ is the number of vertices in the graph $\mathbb{Q}_{n}$ and $s$ is the number of nonisomorphic indecomposable factors of $A=C_{\Omega}(d)$.

Proof. We use the same notation as in the proof of Proposition 3.1. Let $d^{\prime}=\left(d_{1}, \ldots, d_{n-1}\right)$ and assume by induction that $\left|I_{d^{\prime}}\right|=(n-1)-s^{\prime}$, where $s^{\prime}$ is the number of nonisomorphic factors in $A^{\prime}=C_{\Omega^{\prime}}\left(d^{\prime}\right)$. Note that either $\left|I_{d}\right|=\left|I_{d^{\prime}}\right|$ or $\left|I_{d}\right|=\left|I_{d^{\prime}}\right|+1$, (adding the vertex $n$ and passing from $d^{\prime}$ to $d$ we can at most create a new pair $(i, n), i<n$, in $\left.I_{d}\right)$. Therefore we only have to prove that we have in $I_{d}$ a pair $(i, n)$ if and only if $s=s^{\prime}$.

The claim is obvious if $d_{n} \geqslant d_{n-1}$. In fact $(n-1, n) \in I_{d}$ implies $d_{n}=d_{n-1}$ and $s=s^{\prime}$ and conversely. If $d_{n}>d_{n-1}$ then $(i, n) \notin I_{d}$ for every $i$ (cf. $(4.1)^{\prime}$, condition $(-1)$ ), and $s \neq s^{\prime}$ as in $A$ we have factors $E_{n n}$. Assume $d_{n}<d_{n-1}$. Then $s=s^{\prime}$ if and only if adding the vertex $n$ to the construction $C_{\Omega^{\prime}}\left(d^{\prime}\right)$ not all the factors which are of type $E_{q, n-1}$ in $A^{\prime}$ have changed to $E_{q n}$ in $A$; moreover in $A$ there are factors $E_{p n}$ (some $p$ ) and $E_{p, n-1}\left(\right.$ same $p$ ) is not a factor. Therefore $s=s^{\prime}$ is equivalent to saying that the following sets are not empty:

$$
\Theta_{n-1}=\left\{q, q=1, \ldots, n-1, e_{q, n-1}^{A}>0\right\}, \quad \Theta_{n}=\left\{p, p=1, \ldots, n-1, e_{p n}^{A}>0\right\}
$$

and $\Theta_{n-1} \cap \Theta_{\mathrm{n}}=\varnothing$. Let $\bar{q}=\min _{q \in \Theta_{n}} q, \bar{p}=\min _{p \in \Theta_{n-1}} p, i+1=\max (\bar{p}, \bar{q})$. Then the pair $(i, n)$ belongs to $I_{d}$, as follows easily from the interpretation of the conditions defining $I_{d}$ in terms of the construction $C_{\Omega}(d)$ and Lemma 3.2 and conversely.

The importance of this proposition comes from the following results of Happel (cf. [6]) and Sato and Kimura (cf. [9]) (cf. also §6).

Proposition 4.4. (a) $n-s$ equals $\operatorname{dim}\left(L_{d} / \Pi \operatorname{SL}\left(V_{i}\right)\right)$.

(b) $n-s$ equals the number of the codim 1 orbits in $L_{d}$.

5. Classification of the codim 1 orbits in $L_{d}\left(Q_{n}, \Omega\right)$. Let $\Theta_{1}$ be the set of codimension 1 orbits in $L_{d}\left(Q_{n}, \Omega\right)$ and $A=C_{\Omega}(d)$ a representative of the maximal orbit.

THEOREM 5.1. There exists a unique bijective map $\psi: I_{d} \rightarrow \mathcal{O}_{1}\left(\psi(i, j)=O_{i j}=O_{B_{i}}\right.$, $B_{i j} \in L_{d}$ a representative in $O_{i j}$ ) such that:

(i) $N_{i j}^{B_{i j}}=N_{i j}^{A}-1$.

(ii) $N_{u v}^{B_{i}}=N_{u v}^{A}$ for every $(u, v) \in I_{d},(u, v) \neq(i, j)$.

Proof. We first construct the map $\psi$. Let $(i, j) \in I_{d}, s_{\alpha-1} \leqslant i<s_{\alpha}, s_{\beta}<j \leqslant s_{\beta+1}$. Define $\Lambda_{i j}^{(\mathrm{e})}$ to be the set of pairs of indecomposable factors of $A$ of type ( $E_{h k}$, $\left.E_{i+1, j-1}\right)$ such that $h \leqslant i, k \geqslant j$. This set is not empty if and only if $\beta-\alpha$ is odd as we have seen in Remark 4.2. Define $\Lambda_{i j}^{(o)}$ to be the set of pairs of factors in $A$ of type $\left(E_{h, j-1}, E_{i+1, k}\right)$ such that $h \leqslant i, k \geqslant j$. This set is not empty if and only if $\beta-\alpha$ is even. It follows that the set $\Lambda_{i j}=\Lambda_{i j}^{(\mathrm{e})} \cup \Lambda_{i j}^{(\mathrm{o})}$ is not empty and if $\Lambda_{i j}^{(\mathrm{e})} \neq \varnothing$ then $\Lambda_{i j}^{(o)}=\varnothing$. Note that on any pair of indecomposables in $\Lambda_{i j}^{(\mathrm{e})}$ we can perform an elementary degeneration of type (e) and on each pair in $\Lambda_{i j}^{(\text {o) }}$ we can perform an 
elementary degeneration of type (o) (cf. $\S 1$ ). The representation $B_{i j}$ associated via $\psi$ to the pair $(i, j)$ is now defined performing an elementary degeneration of type (e) or (o) on a pair in $\Lambda_{i j}^{(\mathrm{e})}$ or in $\Lambda_{i j}^{(\mathrm{o})}$ for a suitable choice of the indices $h$ and $k$.

Note that if $\beta-\alpha$ is odd, i.e. if $\Lambda_{i j}^{(\mathrm{e})} \neq \varnothing$ and $\Lambda_{i j}^{(o)}=\varnothing$ we cannot specify a priori the type (odd or even) of the factors $E_{h k}, h \leqslant i, k \geqslant j$ as $i \geqslant 1$ and $j \leqslant m$, but we do know that $E_{i+1, j-1}$ is of even type. Similarly if $\beta-\alpha$ is even, we do not know a priori the type (odd or even) of $E_{h, j-1}$ and $E_{i+1, k} h \leqslant i, k \geqslant j$, but we do know that $[i+1, j-1]$ is of odd type. Therefore a priori all the four cases listed in the table at the end of $\S 1$ are possible; it follows that our choice of the integers $h$ and $k$ must be done choosing first the case I, II, III or IV which is the most convenient for our purpose of ending up with a codim 1 orbit (cf. $\S 2$, Column 1 of the table). We simply write $\Lambda$ instead of $\Lambda_{i j}$ and consider the decomposition $\Lambda=\Lambda_{\mathrm{I}} \cup \Lambda_{\mathrm{II}} \cup \Lambda_{\mathrm{III}} \cup \Lambda_{\mathrm{IV}}$, where $\Lambda_{\rho}, \rho=$ I, II, III or IV, is the subset of $\Lambda$ on which the elementary degeneration corresponds to the case $\rho$. Note that if $\Lambda_{\text {II }} \neq \varnothing$ then $\Lambda_{\text {III }}=\varnothing$ as a consequence of Lemma 3.2, and conversely.

We choose the indices $h$ and $k$ according to the following criterion:

If $\Lambda_{\text {IV }} \neq \varnothing$, then among the pairs in $\Lambda_{\text {IV }}$ we choose one with $k-h$ minimum possible.

If $\Lambda_{\text {IV }}=\varnothing$ and $\Lambda_{\text {III }} \neq \varnothing$, then among the pairs in $\Lambda_{\text {III }}$ we choose $k$ minimum possible and $h$ minimum possible.

If $\Lambda_{\text {IV }}=\Lambda_{\text {III }}=\varnothing$ and $\Lambda_{\text {II }} \neq \varnothing$, then among the pairs in $\Lambda_{\text {II }}$ we choose $k$ maximum possible and $h$ maximum possible.

If $\Lambda_{\text {IV }}=\Lambda_{\text {III }}=\Lambda_{\text {II }}=\varnothing$, then $\Lambda_{\text {I }} \neq \varnothing$ and we choose $k-h$ maximum possible.

Note that the choice of the indices $h$ and $k$ is in any case uniquely determined, as a consequence of Lemma 3.2. With the choice of $h$ and $k$ just specified we will have either

(1) $\Lambda_{i j}=\Lambda_{i j}^{(\mathrm{e})} ; A=\bar{A} \oplus E_{h k} \oplus E_{i+1, j-1} ; B_{i j}=\bar{A} \oplus E_{h, j-1} \oplus E_{i+1, k}$ or

(2) $\Lambda_{i j}=\Lambda_{i j}^{(o)} ; A=\bar{A} \oplus E_{h, j-1} \oplus E_{i+1, k} ; B_{i j}=\bar{A} \oplus E_{h, k} \oplus E_{i+1, j-1}$

and from now on we will use the notations of $\$ 2$ (codimension formulas), assuming $B=B_{i j}$ and $r=i+1, t=j-1$.

We have $\operatorname{codim}_{L_{d}} \bar{O}_{i j}=1$; in fact, according to Proposition 2.5 we have $e_{p q}^{\bar{A}}=0$ for every $(p, q) \in H(h, r, t, k)$ as a consequence of the priorities given to the cases IV, III, II, I and the choice of the indices $h, k$ (cf. $\$ 2$, Column 1 of the table). The map $\psi$ is injective. In fact if $(i, j),\left(i^{\prime}, j^{\prime}\right) \in I_{d}$ and $(i, j) \neq\left(i^{\prime}, j^{\prime}\right)$ then $i \neq i^{\prime}, j \neq j^{\prime}$. It follows that $O_{i j} \neq O_{i^{\prime} j^{\prime}}$ as $B_{i j}$ and $B_{i^{\prime} j^{\prime}}$ are both obtained from $A$ by performing an elementary degeneration on distinct pairs of indecomposables. From Propositions 4.3 and 4.4 it now follows that $\psi$ is bijective.

Proof of (i). It follows from the fact that the pair $(i, j)=(r-1, t+1)$ is listed in Column 2 of the table in $\S 2$.

Proof of (ii). By contradiction assume $N_{u v}^{B_{i}}=N_{u v}^{A}-1$ for some $(u, v) \in I_{d}$. Consider first the case when $B_{i j} \in O_{i j}=\psi(i, j)$ is obtained from $A$ via an elementary degeneration of type (e), i.e. assume the set $\Lambda_{i j}^{(\mathrm{e})} \neq \varnothing$, the degeneration belonging to one of the cases IV, III, II or I according to the rule given by $\psi$. The condition $N_{u v}^{B_{i}}=N_{u v}^{A}-1$ implies that $(u, v)$ is one of the pairs listed in Column 2 of the table 
on the row corresponding to the case we are dealing with. On the other side the condition $(u, v) \in I_{d}$ implies that we have the representation $B_{u v} \in O_{u v}=\psi(u, v)$ and therefore in $A$ there is either $E_{u+1, v-1} \oplus E_{\lambda, \mu}$ (if the degeneration which gives rise to $B_{u v}$ is of type (e)), or $E_{\lambda, v-1}+E_{u+1, \mu}$ (if the degeneration is of type (o)) and in any case $\lambda \leqslant u<i, \mu \geqslant v>j$. In the first case $(u+1, v-1)$ is a pair in $H(h, r, t, k)$ (compare Columns 1 and 2 ); in the second case either $(\lambda, v-1)$ or $(u+1, \mu)$ is in $H(h, r, t, k)$. Therefore we get a contradiction to the assumption $\operatorname{codim}_{L_{d}} \bar{O}_{i j}=1$. Suppose now that $B_{i j}$ is obtained via a degeneration of type (o), i.e. $\Lambda_{i j}^{(o)} \neq \varnothing$. Again in $A$ either we have the factors $E_{u+1, v-1} \oplus E_{\lambda, \mu}$ (if $B_{u v}$ is obtained from $A$ via a degeneration of type (e)), or $E_{\lambda, v-1} \oplus E_{u+1, \mu}$ (if the degeneration is of type (o)) and always $\lambda \leqslant u<i, \mu \geqslant v>j$. It follows that in $A=C_{\Omega}(d)$ we have factors $E_{z w}$ with $[z, w] \supsetneq[i, j]$ which is a contradiction to the conditions $(i, j) \in I_{d}$ and $B_{i j}$ obtained with a degeneration of type (o) (cf. Remark 4.2).

EXAMPLE.

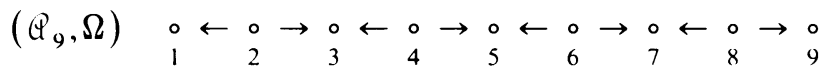

$d=(4,3,4,4,5,3,2,1,2), I_{d}=\{(2,8),(3,4),(4,7)\}$,

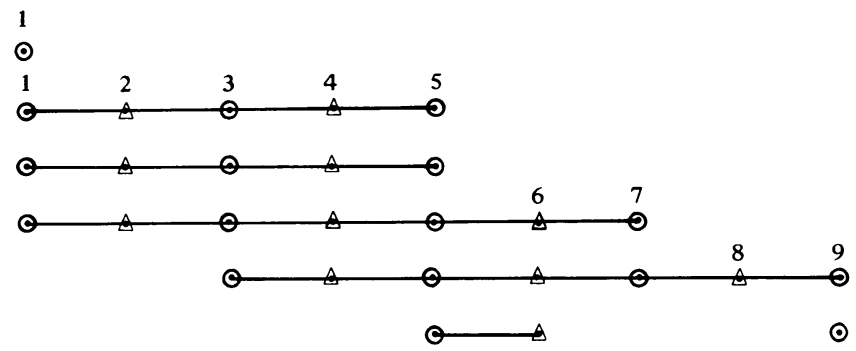

$A=C_{\Omega}(d)=E_{1,1} \oplus 2 E_{1.5} \oplus E_{1,7} \oplus E_{3,9} \oplus E_{5,6} \oplus E_{9,9}, n-s=3$, $B_{2,8}$ is obtained performing the operation $E_{1,7} \oplus E_{3,9} \mapsto E_{3,7} \oplus E_{1.9}$, $B_{4.7}$ is obtained performing the operation $E_{3,9} \oplus E_{5.6} \mapsto E_{3,6} \oplus E_{5.9}$, $B_{3.4}$ is obtained performing the operation $E_{1.5} \mapsto E_{1.3} \oplus E_{4.5}$.

6. The ring of semi-invariant polynomials $\Re_{d}\left(\bigotimes_{n}, \Omega\right)$. We recall first some known facts. Let $K$ be an algebraically closed field and $\rho$ a rational representation of a connected algebraic group $G$ on a finite-dimensional vector space $V$ over $K$. Then $G$ acts on the polynomial ring $K[V]$ of $V$ via the action

$$
f^{g}(v)=f\left(\rho\left(g^{-1}\right) v\right), \quad g \in G .
$$

A polynomial $f \in K[V]$ is said to be semi-invariant (or relative invariant) if

$$
f^{g}=\chi(g) \cdot f \quad(\chi \text { a character of } G) .
$$

The ring of semi-invariants of the representation $(G, \rho, V)$ is, by definition, the ring generated by the semi-invariant polynomials. 
$(G, \rho, V)$ is called prehomogeneous if there is an open dense orbit $O_{\max } \subset V$. In this case it has been proved by M. Sato and T. Kimura (cf. [9]) that the reduced equations $f_{i}=0$ of the codim 1 components in $V \backslash O_{\max }$ are such that the $f_{i}$ 's are an algebraically independent system of semi-invariant polynomials which generate the ring of semi-invariants.

Consider now, for a given dimension $d=\left(d_{1}, \ldots, d_{n}\right)$, the variety $L_{d}\left(Q_{n}, \Omega\right)=$ $\Pi_{l \in \Gamma_{1}} \operatorname{Hom}_{K}\left(V_{i(l)}, V_{f(l)}\right)$ defined in $\S 0$ and the corresponding action $\rho$ of the group $G_{d}$. Then $\left(G_{d}, \rho, L_{d}\right)$ is prehomogeneous and the codim 1 components in $L_{d} \backslash O_{\max }$ are exactly the closures of the codim 1 orbits in $L_{d}$ (it follows from the fact that this action has a finite number of orbits).

In view of the mentioned theorem of Sato and Kimura, we want to compute for each codim 1 orbit in $L_{d}$ the reduced equation of its closure.

If we fix bases in the vector spaces $V_{i}$ 's then $L_{d}$ is identified to the set of $(n-1)$-tuples of matrices $X=\left(X_{1}, X_{2}, \ldots, X_{n-1}\right)$, where $X_{t}$ is a $d_{t} \times d_{t+1}$ matrix if in the given orientation $\Omega$ the edge $l=[t, t+1]$ is such that $i(l)=t+1, f(l)=t$, or a $d_{t+1} \times d_{t}$ matrix if $i(l)=t, f(l)=t+1$. Then, for every $i<j, i, j=1, \ldots, n$, we can write the matrix $Y_{i j}$ corresponding to the map $\varphi_{i j}^{X}$ (cf. $\left.\S 1\right)$ in the induced bases.

Assume $s_{\alpha-1} \leqslant i<s_{\alpha}, s_{\beta}<j \leqslant s_{\beta+1}$, and $s_{\alpha}$ a sink and let $\bar{X}_{i, s_{\alpha}}, \bar{X}_{s_{\alpha}, s_{\alpha+1}}, \ldots, \bar{X}_{s_{\beta}, j}$ be the product of the $X_{t}$ 's between $i$ and $s_{\alpha}, s_{\alpha}$ and $s_{\alpha+1}$ etc., i.e., $\bar{X}_{i, s_{\alpha}}=X_{s_{\alpha}-1} \circ \cdots$ $\circ X_{i+1} \circ X_{i}, \bar{X}_{s_{\alpha}, s_{\alpha+1}}=X_{s_{\alpha}} \circ X_{s_{\alpha}+1} \circ \cdots \circ X_{s_{\alpha+1}-1}$, etc. Then we have

$$
Y_{i j}=\left[\begin{array}{cccccc}
\bar{X}_{i, s_{\alpha}} & -\bar{X}_{s_{\alpha}, s_{\alpha+1}} & 0 & \cdots & 0 & 0 \\
0 & \bar{X}_{s_{\alpha+1}, s_{\alpha+2}} & -\bar{X}_{s_{\alpha+2}, s_{\alpha+3}} & \cdots & 0 & 0 \\
0 & 0 & \bar{X}_{s_{\alpha+3}, s_{\alpha+4}} & \cdots & & \\
0 & 0 & 0 & & \\
\vdots & \vdots & \vdots & & \\
0 & 0 & 0 & & \pm \bar{X}_{s_{\beta}, j}
\end{array}\right] .
$$

A similar expression holds for $Y_{i j}$ in case $s_{\alpha}$ is a source.

Assume now $(i, j) \in I_{d}$, then $Y_{i j}$ is a square matrix and we define

$$
D_{i j}=\operatorname{Det}\left(Y_{i j}\right) \quad\left((i, j) \in I_{d}\right) .
$$

These are the polynomials to which we refer in $\S 0$ and, in particular, in Theorem 0.1 .

Proof of Theorem $0.1 . D_{i j}$ is not identically zero as it is not zero on the open orbit $O_{\max }^{d} \subset L_{d} . D_{i j}$ vanishes on $\bar{O}_{i j}$ as a consequence of Theorem 5.1(i), and does not vanish on any other codim 1 orbit of $L_{d}$ as a consequence of Theorem 5.1(ii). We want to prove that $D_{i j}=0$ is the reduced equation of the subvariety $\bar{O}_{i j}$. The proof is now the same as the one given in [1]. We repeat it for the convenience of the reader.

Assume $D_{i j}=(f)^{t}$ for some polynomial function $f$ on $L_{d}$ and $f=0$ the reduced equation of $\bar{O}_{i j}$. Then $f$ is a semi-invariant polynomial. Let $T^{n}=\prod_{i=1}^{n} G_{m}^{(i)}, G_{m}^{(i)}$ a copy of the multiplicative group $G_{m}$ of $K . T^{n}$ acts on the matrices $X_{i}$ 's and therefore on the $\bar{X}$ 's and on $Y_{i j}$. Explicitly we have for any $a=\left(a_{1}, \ldots, a_{n}\right) \in T^{n},(i, j) \in I_{d}$, $a D_{i j}=\left(a_{i}\right)^{-d_{i}}\left(a_{s_{\alpha}}\right)^{d_{s_{\alpha}}} \cdots\left(a_{j}\right)^{ \pm d_{j}} D_{i j}$, where the sign \pm corresponds to the fact that 
$s_{\beta}$ can be either a source or a sink (we always assume for simphity that $s_{x x}$ is a sink). It follows from the assumption $D_{i j}=(f)^{t}$ that

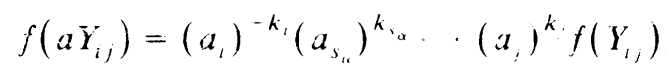

$k_{i}, k_{s_{\alpha}}, \ldots, k_{i}$ posiive integers and $i_{t}-i k_{l}, \ldots, d_{j}=i k_{j}$. If $d_{1}=1$ then $t=1$ and there is nothing to be proved. If $d,>1$ and the field $K$ is algebraically closed and of chiar 0 we may chuose $a_{j}$ to be a primitive $d_{j}$ root of 1 and all the other coordinates of $a$ equal to 1 . Then $a \in \Pi_{r=1}^{n} \operatorname{SL}\left(d_{r}, K\right)$ and therefore $\left(u_{j}\right)^{k}=1$, i.e. $d$, divides $k_{\text {, }}$ and $t=1$. If char $K \neq 0$ one may substitute this with an argument on formal invariants. In fact if $f^{g}=f$ for any $g \in \operatorname{SL}\left(d_{j}, K\right)$ the same identity holds with $g \in \mathrm{SL}\left(d_{j}, A\right)$ and $A$ any $K$-algebra; in particular we can take $A=K[z] /\left(z^{d_{j}}-1\right)$ and $a_{j}$ the class $\bar{z}$ of $z$. The same argument as before holds, and Theorem 0.1 follows as a consequence of the mentioned theorem of Sato and Kimura (cf. [9]). If $K$ is not algebraically closed but infinite, Theorem 0.1 still holds. In fact the semi-invariant polynomials $D_{i j}$ have coefficients in $K$ and if $\bar{K}$ denotes the algebraic closure of $K$ then $G_{d}=\Pi \mathrm{GL}\left(d_{r}, K\right)$ is dense in $\bar{G}_{d}=\Pi \mathrm{GL}\left(d_{r}, \bar{K}\right)$.

7. Reflections and semi-invariants. We start our discussion considering the oriented graph

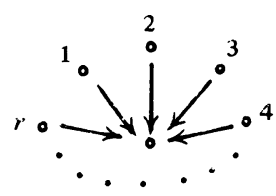

and the variety $L$ of all its representations of a given dimension $\alpha=\left(h ; h_{1}\right.$, $\left.h_{1}, \ldots, h_{r}\right)$, i.e. we consider vector spaces $V_{i}, V(i=1, \ldots, r)$, over $K, \operatorname{dim} V_{i}=h_{l}$, $\operatorname{dim} V=h$, and fix bases in $V_{i}, V$. Then $L=\prod_{i=1}^{r} \operatorname{Hom}_{K}\left(V_{i}, V\right) \simeq\left\{\left(X_{1}, X_{2}, \ldots, X_{r}\right)\right\}$, where the $X_{i}$ 's are $h \times h_{i}$ matrices:

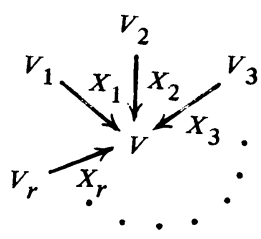

Let $Q=K[L]=K\left[X_{1}, \ldots, X_{r}\right]$ be the ring of polynomials of $L$. Assume now that $h<h_{1}+h_{2}+\cdots+h_{r}$ and consider the matrix $X:=\left[X_{1} X_{2} \cdots X_{r}\right]$. We denote by $\left[\begin{array}{llll}i_{1} & i_{2} & \cdots & i_{h}\end{array}\right]_{X}$ the determinant of the $h \times h$ minor relative to the column indices $i_{1}$, $i_{2}, \ldots, i_{h}\left(i_{1}<i_{2}<\cdots<i_{h}\right)$. The special linear group $\operatorname{SL}(V)=\operatorname{SL}(h, K)$ acts on 
$L$ and we denote by $\mathbb{A}^{\mathrm{SL}(h, K)} \subset \mathbb{A}^{\mathrm{S}}$ the subring of polynomials which are invariant under $\operatorname{SL}(h, K)$. Then we have

$$
\mathcal{H}^{\mathrm{SL}(h, K)}=K\left[\left[i_{1} i_{2} \cdots i_{h}\right]_{X}\right]
$$

The group $G:=\prod G L\left(V_{i}\right)=\prod G L\left(h_{1}, K\right)$ acts on $L$ and the induced action on $u^{(S L}(h, K)$ is the one induced on $\left[i_{1} \cdots i_{h}\right]_{X}$ by the right multiplication

$$
X \cdot\left[\begin{array}{cccc}
A_{1} & 0 & \cdots & 0 \\
0 & A_{2} & . & . \\
\vdots & \vdots & & \vdots \\
0 & 0 & & A_{r}
\end{array}\right], \quad A_{l} \in G L\left(h_{l}, K\right) .
$$

We perform now a simple reflection with respect to the central vertex of our graph and consider the reflected dimension $\alpha^{\prime}$ corresponding to $\alpha$. We get the following picture, where we use similar notations to the one introduced before:
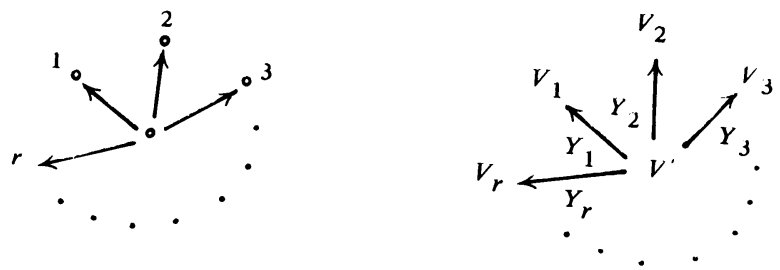

$$
\begin{gathered}
\alpha^{\prime}=\left(h^{\prime} ; h_{1}, h_{2}, \ldots, h_{r}\right), \quad h^{\prime}=h_{1}+\cdots+h,-h, \\
\operatorname{dim} V^{\prime}=h^{\prime}, \quad Y_{i} \text { an } h_{i} \times h^{\prime} \text { matrix, } \\
L^{\prime}=\prod \operatorname{Hom}_{K}\left(V^{\prime}, V_{i}\right)=\left\{\left(Y_{1}, \ldots, Y_{r}\right)\right\}, \quad Q^{\prime}=K\left[L^{\prime}\right], \\
Y=\left[\begin{array}{c}
Y_{1} \\
Y_{2} \\
Y_{3} \\
\vdots \\
Y_{r}
\end{array}\right], \quad \mathbb{Q}^{\prime} \operatorname{SL}\left(h^{\prime} K\right)=K\left[\left[j_{1}, \ldots, j_{h}\right]_{Y}\right] .
\end{gathered}
$$

The action of $G$ on $\mathcal{Q}^{\prime} \mathrm{SL}\left(h^{\prime}, K\right)$ is induced by the action of $G$ on $Y$ given by the left multiplication.

Proposition 7.1. The murphism $\mathbb{Q}: \mathbb{Q}^{\prime S L}\left(h^{\prime}, K\right) \rightarrow \mathbb{Q}^{\mathrm{SL}(h, K)}$ given by $\left[j_{1} \cdots j_{h}\right]_{Y} \rightarrow$ $\varepsilon_{\sigma}\left[i_{1} \cdots i_{h}\right]_{X}$, where $\sigma=\left(j_{1}, \ldots, j_{h^{\prime}}, i_{1}, \ldots, i_{h}\right)$ is a permutation of the indices $(1$, $\left.2, \ldots, h+h^{\prime}\right)$ and $\varepsilon_{\sigma}$ its sign, is an isomorphism compatible with the actlon of the group $G$. 
Proof. We give only a sketch, as the argument is completely standard. Let $m=h+h^{\prime}$ and let $W$ be a vector space over $K$ of dimension $m$. We fix a basis $e_{1}$, $e_{2}, \ldots, e_{m}$ in $W$ and an explicit identification $\wedge{ }^{m} W \stackrel{\sim}{\rightarrow} K$ given by $e_{1} \wedge e_{2} \wedge \cdots \wedge$ $e_{m} \mapsto 1$, moreover we consider the canonical pairings

$$
\begin{aligned}
& h \quad h \\
& \wedge W^{*} \times \wedge W \rightarrow K, \quad\left(\varphi_{1} \wedge \cdots \wedge \varphi_{h}, w_{1} \wedge \cdots \wedge w_{h}\right) \mapsto \operatorname{det}\left[\left\langle\varphi_{i}, w_{j}\right\rangle\right], \\
& \stackrel{h}{\wedge} W \times \stackrel{h}{\prime}^{\prime} W \rightarrow \stackrel{m}{\wedge} W, \quad\left(w_{1} \wedge \cdots \wedge w_{h}, w_{1}^{\prime} \wedge \cdots \wedge w_{h^{\prime}}^{\prime}\right) \\
& \mapsto w_{1} \wedge \cdots \wedge w_{h} \wedge w_{1}^{\prime} \wedge \cdots \wedge w_{h^{\prime}}^{\prime}
\end{aligned}
$$

Then we have an $\operatorname{SL}(W)$-equivariant isomorphism

$$
\tilde{\mathbb{Q}}: \stackrel{h}{\wedge} \stackrel{\sim}{\sim} W^{*} \stackrel{h^{\prime}}{\wedge} \wedge W
$$

which sends the affine cone over the Grassmann variety $G_{h, m}\left(W^{*}\right)$ to the affine cone over $G_{h^{\prime}, m}(W)$ and induces the given $Q$.

Proposition 7.1 gives an explicit construction of the first isomorphism given in [7] (cf. formula (2.19) in the corollary to Proposition 2.1, p. 77). In fact when we apply a reflection relative to an admissible vertex of an oriented graph (a vertex which is either a source or a sink), the part of the graph which is involved is of the type assumed at the beginning of this section.

We reformulate the content of the mentioned corollary of [7] in the case of the graph $Q_{n}$, using the notation introduced in $\$ \S 1-6$.

Let $\Omega$ and $\Omega^{\prime}$ be two orientations of $\mathbb{Q}_{n}$ and assume that $\Omega^{\prime}$ is obtained from $\Omega$ via a reflection at the admissible vertex $t$. Let $d=\left(d_{1}, \ldots, d_{t}, \ldots, d_{n}\right)$ be a given dimension for the representations of $\left(Q_{n}, \Omega\right)$ and $d^{\prime}=\left(d_{1}, \ldots, d_{t}^{\prime}, \ldots, d_{n}\right), d_{t}^{\prime}=d_{t-1}$ $+d_{t+1}-d_{t}$, a dimension for the representations of $\left(\mathbb{Q}_{n}, \Omega^{\prime}\right)$. Then we have

Proposition 7.2 (V. KAC). 1. $\mathscr{R}_{d}\left(\mathbb{Q}_{n}, \Omega\right) \stackrel{\theta}{\leftrightarrow} \mathscr{R}_{d^{\prime}}\left(\mathbb{Q}_{n}, \Omega^{\prime}\right)$ if $d_{t}^{\prime}>0$,

2. $\Re_{d}\left(\Theta_{n}, \Omega\right) \stackrel{\sim}{\leftrightarrow} \mathscr{R}_{d^{\prime}}\left(Q_{n}, \Omega^{\prime}\right) \otimes_{K} K\left[D_{t-1, t+1}\right]$ if $d_{t}^{\prime}=0$,

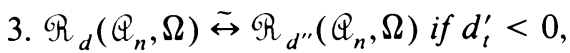

where if $d_{t}^{\prime}=d_{t-1}+d_{t+1}-d_{t}=0$ then $(t-1, t+1) \in I_{d}(\Omega)$ and $D_{t-1, t+1}$ is the corresponding determinant defined in $\S 6$; if $d_{t}^{\prime}<0, d^{\prime \prime}=\left(d_{1}, \ldots, d_{t-1}, 0, d_{t+1}, \ldots, d_{n}\right)$.

Let $\Omega, \Omega^{\prime}, d$ and $d^{\prime}$ be as before and let $d_{t}^{\prime}>0$. In order to prove Theorem 0.1 by deducing it from the explicit construction of the ring of semi-invariants in the equioriented case (cf. [1]), one should directly prove, among others, the following proposition. Let $I_{d}(\Omega)$ and $I_{d^{\prime}}\left(\Omega^{\prime}\right)$ be the sets defined in $\S 4$, and $D_{i j}(\Omega)$, $(i, j) \in I_{d}(\Omega), D_{r s}^{\prime}\left(\Omega^{\prime}\right), \quad(r, s) \in I_{d^{\prime}}\left(\Omega^{\prime}\right)$, the proposed generators respectively for $\Re_{d}\left(Q_{n}, \Omega\right)$ and $\Re_{d^{\prime}}\left(Q_{n}, \Omega^{\prime}\right)$.

Proposition 7.3. $\theta\left(D_{i j}(\Omega)\right)=\lambda \cdot D_{r s}^{\prime}\left(\Omega^{\prime}\right)$ for every $(i, j) \in I_{d}(\Omega)$ and suitable $(r$, $s) \in I_{d^{\prime}}\left(\Omega^{\prime}\right), \lambda$ a scalar. 
This proposition follows immediately from Proposition 7.2 and Theorem 0.1 once we prove this last theorem directly from Theorem 5.1. A direct proof can be done and involves explicit computations of the determinants.

We want to give an idea of this type of computation. In order to simplify our notations we consider the following special case (which in any case contains all the ingredients of the general case). Assume we have the Dynkin diagram $Q_{7}$ oriented in such a way that all its vertices are either a source or a sink:

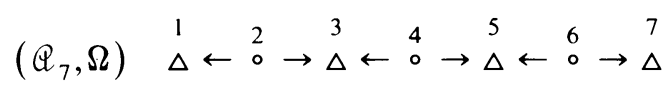

Let $d=\left(d_{1}, d_{2}, \ldots, d_{7}\right)$ and

$$
L_{d}=\left\{\cdot \stackrel{x_{1}}{\leftarrow} \cdot \stackrel{x_{2}}{\rightarrow} \cdot \stackrel{X_{3}}{\leftarrow} \cdot \stackrel{x_{4}}{\rightarrow} \cdot \stackrel{X_{5}}{\leftarrow} \cdot \stackrel{x_{6}}{\rightarrow} \cdot\right\}
$$

assume moreover that $(1,7) \in I_{d}(\Omega)$. If we use the reflection at the vertex 4 the corresponding situation is:

$$
\begin{aligned}
& \left(Q_{7}, \Omega^{\prime}\right) \quad \stackrel{1}{\Delta} \leftarrow \stackrel{2}{\circ} \rightarrow \stackrel{3}{\rightarrow} \rightarrow{ }^{4} \leftarrow \leftarrow^{5} \leftarrow \stackrel{6}{\circ} \rightarrow{ }^{7} \\
& d^{\prime}=\left(d_{1}, d_{2}, d_{3}, d_{4}^{\prime}, d_{5}, d_{6}, d_{7}\right), \quad d_{4}^{\prime}=d_{3}+d_{5}-d_{4}>0, \\
& L_{d^{\prime}}=\left\{\cdot \stackrel{X_{1}}{\leftarrow} \cdot \stackrel{X_{2}}{\rightarrow} \cdot \stackrel{Y_{3}}{\rightarrow} \cdot \stackrel{Y_{4}}{\leftarrow} \cdot \stackrel{X_{5}}{\leftarrow} \cdot \stackrel{X_{6}}{\rightarrow} \cdot\right\} .
\end{aligned}
$$

Again $(1,7) \in I_{d^{\prime}}\left(\Omega^{\prime}\right)$ and up to signs we have

$$
D_{1,7}(\Omega)=\left[\begin{array}{ccc}
X_{1} & 0 & 0 \\
X_{2} & X_{3} & 0 \\
0 & X_{4} & X_{5} \\
0 & 0 & X_{6}
\end{array}\right], \quad D_{1,7}^{\prime}\left(\Omega^{\prime}\right)=\left[\begin{array}{cc}
X_{1} & 0 \\
Y_{3} X_{2} & Y_{4} X_{5} \\
0 & X_{6}
\end{array}\right]
$$

The isomorphism $\mathbb{Q}$ of Proposition 7.2, 1, is induced by the correspondence between the maximal minors of the two matrices $Y=\left[Y_{3} Y_{4}\right],\left(\mathrm{a} d_{4}^{\prime} \times\left(d_{3}+d_{5}\right)\right.$ matrix $)$ and $X=\left[\begin{array}{l}X_{3} \\ X_{4}\end{array}\right]\left(\mathrm{a}\left(d_{3}+d_{5}\right) \times d_{4}\right.$ matrix $)$, and is the identity on all the other matrix variables. In the remaining part we will use the following notations: for every matrix $Z$ we denote by $|Z|_{j_{1} \cdots j_{s}}^{i_{1} \ldots i_{s}}$ the determinant of the minor relative to the columns $i_{1}<i_{2}<\cdots<i_{s}$ and to the rows $j_{1}<j_{2}<\cdots<j_{s}$. We will omit the upper indices in case we have to take all the column indices and similarly for the rows.

In order to prove that $\theta\left(D_{1,7}^{\prime}\left(\Omega^{\prime}\right)\right)=\lambda D_{1,7}(\Omega)$ we compute both determinants using Laplace expansion. For $D_{1,7}^{\prime}\left(\Omega^{\prime}\right)$ we use Laplace expansion relative to the rows of $\left[\begin{array}{ll}Y_{3} X_{2} & Y_{4} X_{5}\end{array}\right]$ and we note that

$$
\left[\begin{array}{ll}
Y_{3} X_{2} & Y_{4} X_{5}
\end{array}\right]=\left[Y_{3} Y_{4}\right] \cdot\left[\begin{array}{cc}
X_{2} & 0 \\
0 & X_{5}
\end{array}\right]
$$


It follows that

$$
\begin{aligned}
& \Gamma_{1,7}^{\prime}\left(\Omega^{\prime}\right)=\sum \pm \|\left.\left[\begin{array}{ll}
Y_{3} X_{2} & Y_{4} X_{5}
\end{array}\right]\right|^{\prime \prime} d_{d_{4}}\left|\left[\begin{array}{cc}
X_{1} & 0 \\
0 & X_{6}
\end{array}\right]\right|^{\left(/ 1 \cdots d_{1 / 4}\right)^{*}} \\
& =\sum \pm\left|\left[\begin{array}{ll}
Y_{3} & Y_{4}
\end{array}\right]\right|^{s_{1} \cdots s_{d_{4}}} \cdot\left|\left[\begin{array}{cc}
X_{2} & 0 \\
0 & X_{5}
\end{array}\right]\right|_{s_{1} \cdots s_{d_{4}}}^{j_{1} \cdots j_{d_{4}}} \cdot\left|\left[\begin{array}{cc}
X_{1} & 0 \\
0 & X_{6}
\end{array}\right]\right|^{\left(j_{1} \cdots j_{d_{4}}\right)^{*}} \text {, }
\end{aligned}
$$

where $\left\{d_{1} \cdots j_{d_{4}^{\prime}}\right\} \subset\left\{1,2, \ldots, d_{2}+d_{6}\right\}$ and $\left(j_{1} \cdots j_{d_{4}^{\prime}}\right)^{*}$ denotes the complement of $\left\{j_{1}, \ldots, j_{d^{\prime}}\right\}$ in $\left\{1.2, \ldots, d_{2}+d_{6}\right\}$.

$$
\theta\left(\Gamma_{1,7}^{\prime}\left(\Omega^{\prime}\right)\right)=\sum \pm \mid\left[\begin{array}{l}
X_{3} \\
X_{4}
\end{array} \|_{\left(s_{1} \cdots s_{d_{4}}\right)^{*}} \cdot\left|\left[\begin{array}{cc}
X_{2} & 0 \\
0 & X_{5}
\end{array}\right]\right|_{s_{1} \cdots s_{d_{4}}}^{j_{1} \cdots d_{d_{4}}} \cdot\left|\left[\begin{array}{cc}
X_{1} & 0 \\
0 & X_{6}
\end{array}\right]\right|^{\left(j_{1} \cdots d_{d_{4}} *^{*}\right.} .\right.
$$

where $\left(s_{1} \cdots s_{d_{4}^{\prime}}\right)^{*}$ is the complement of $\left\{s_{1} \ldots s_{d_{4}^{\prime}}\right\}$ in $\left\{1,2 \ldots d_{3}+d_{5}\right\}$. For $D_{1,7}(\Omega)$ we use the Laplace expansion relative to the columns of

$$
\left[\begin{array}{c}
0 \\
X_{3} \\
X_{4} \\
0
\end{array}\right]
$$

and we end up with the expression $\theta\left(D_{1.7}^{\prime}\left(\Omega^{\prime}\right)\right)$ (up to signs), once we notice that a nonzero minor in

$$
\left[\begin{array}{c}
0 \\
X_{3} \\
X_{4} \\
0
\end{array}\right]
$$

must be taken from $\left[\begin{array}{l}X_{3} \\ X_{4}\end{array}\right]$ and therefore its complement is formed taking all the rows relative to

$$
\left[\begin{array}{cc}
X_{1} & 0 \\
0 & X_{6}
\end{array}\right]
$$

and some rows (the complementary ones) relative to

$$
\left[\begin{array}{cc}
X_{2} & 0 \\
0 & X_{5}
\end{array}\right]
$$

\section{REFERENCES}

1. S. Abeasis, On the ring of semi-invariants for the representations of an equioriented quiver of type $\mathcal{Q}_{n}$, Rull. Un. Mat. Ital. (to appear).

2. S. Abeasis and A. Del Fra. Degenerations for the representations of a quiver of type $Q_{n}, \mathrm{~J}$. Algebra (to appear).

3. I. N. Bernstein, I. M. Gelfand and V. A. Ponomarev, Coxeter functors and Gabriel's theorem, Russian Math. Surveys 28 (1973), 17-32.

4. V. Dlab and G. M. Ringel, Indecomposable representations of graphs and algebras, Mem. Amer. Math. Soc. No. 173 (1976), 1-57. 
5 P. Gabriel, Représentations indécomposables, Sém. Bourbaki, no. 444, 1973/1974. pp. 1-27.

6. I) Happel. Relative invariants and subgeneric orhits of quivers of finite and tame type (to appear;

7. V. Kac. Infinite root svstems. revresentations of graphs and invarian theory. Invent. Miath. 56 (1980), $57-92$

8. Infinite root svstems, representations of graphs and invariant theory. II (to appeari

9. M. Sato and T Kimura, A classification of irreducible pro-homogeneous vector spaces and their relative invariants. Nagnva Math I. 65 (1977), !- 55.

Istituto Matematico. II Universiti di Roma, Roma, Itai.y (Current address)

Department of Mathematics. Massachusftts Institute of Technology, Chmbridge. MasSACHUSFTTS $\cap 2139$ 